\title{
Development and Assessment of a Public Health Alert Delivered through a Community Health Information Exchange
}

\author{
Roland Gamache $^{1,3}$, Kevin C. Stevens ${ }^{2}$, Rico Merriwether ${ }^{3}$, Brian E. Dixon ${ }^{3}$, Shaun
} Grannis ${ }^{1,3}$

\author{
${ }^{1}$ Indiana University School of Medicine \\ ${ }^{2}$ Marion County Health Department, Indiana \\ ${ }^{3}$ Regenstrief Institute
}

\begin{abstract}
Timely communication of information to health care providers during a public health event can improve overall response to such events. However, current methods for sending information to providers are inefficient and costly. Local health departments have traditionally used labor-intensive, mail-based processes to send public health alerts to the provider community. This article describes a novel approach for delivering public health alerts to providers by leveraging an electronic clinical messaging system within the context of a health information exchange. Alerts included notifications related to the 2009 H1N1 flu epidemic, a syphilis outbreak, and local rabies exposure. We describe the process for sending electronic public health alerts and the estimated impact on efficiency and cost effectiveness.
\end{abstract}

Keywords: public health alerts, health information exchange, syndromic surveillance, clinical messaging, H1N1 flu, broadcast alert

\section{Introduction}

A major challenge for public health is facilitating timely communication of information between public health agencies and health care providers. For example, the underreporting of many public health conditions can make accurate surveillance difficult. ${ }^{[1]}$ Conversely, public health professionals find it difficult to let physicians, nurses, and other front line health care workers know that an outbreak may be occurring in their region. A high-priority public health message in the form of an alert is commonly used by public health agencies to share important information with providers.[2] For local health departments, these alerts are typically paper-based, delivered through postal mail, and they target a broad range of health care providers in a specific geographic region (e.g., zip code). Although Health Alert Networks (HANs) have utilized electronic alerts for several years, HANs are often state-run networks that use workflows optimized for non-clinical providers and may reach a limited set of clinical recipients, typically focusing on Sentinel Physicians. [3, 4]

Current paper-based alerting processes have inherent constraints that limit their effectiveness. First, it may take 1 to 4 days for a message sent by the United States Postal Service to reach the 


\section{Development and Assessment of a Public Health Alert Delivered through a Community Health Information Exchange}

physician and additional time to open the mail. Second, the delivery of the mail item is not verified, so it is uncertain if the physician actually receives the message. Finally, traditional sources for physician contact information such as state licensing boards may be incomplete or out-of-date. These issues occur in part because physicians may provide a home or other nonclinical address, or may change practice location after license registration. Consequently, the physician may never receive the alert, receive a delayed alert, or receive the alert in a location where the information may not impact the clinician's decision-making (e.g., the following day when he or she is at the clinical practice site).

The use of a health information exchange (HIE) to deliver public health alerts may help to mitigate these challenges. HIE organizations facilitate the sharing of clinical and administrative health care data among health care institutions, providers, and data repositories. [5] An HIE is an organized entity, often a legal corporation, that specializes in facilitating electronic exchange of health information among a diverse group of often competing health care system stakeholders, including hospitals, laboratories, and physician practices.[6,7] A recent survey reported that as many as one-third of community HIEs may involve data exchange with public health agencies.[8]

We hypothesize that public health agencies may more efficiently communicate with health care providers if HIE infrastructure is leveraged to deliver public health provider alerts. This hypothesis is supported because a core competency of HIEs is to assure that clinical information reaches the appropriate provider at the appropriate place and time, and they often ensure the information is received and utilized. Transmitting public health alert messages can be managed in a fashion nearly identical to clinical information messages, and their receipt can be verified. Finally, electronic public health alerts sent to a provider at their place of practice and incorporated into their routine work processes may increase the likelihood that the information will impact clinical decisions.

With 40 years of experience as a medical informatics research organization Regenstrief researchers have demonstrated that an HIE can be leveraged to support and improve core functions of public health, such as surveillance. [9-11] We have also shown that alerts and reminders incorporated into electronic health record systems can affect clinical decision-making. [12-14] Thus, it should be feasible to leverage an HIE in a similar fashion to deliver alerts and reminders to broad physician cohorts, beyond the scope of one organization and one electronic health record (EHR) system.

This paper describes our initial experiences using an HIE to deliver electronic public health alerts to clinicians. We present a novel framework for alerting community physicians about emerging public health threats while incurring minimal changes to clinical workflow, and we illustrate the framework with real-world scenarios in which the local health department utilized the HIE. Additionally, we discuss draft measures to support evaluating the effectiveness of this framework for public health alert delivery as compared to delivery by current methods. 


\section{Development and Assessment of a Public Health Alert Delivered through a}

Community Health Information Exchange

\section{Methods}

\section{Clinical Message Overview}

In our context, the term "clinical messaging" describes the delivery of clinical results to physician offices using one of three transmission methods: a) direct import into an EHR system, b) electronic push to a secure, web-based portal, or c) facsimile (fax) transmission. Designed and developed at the Regenstrief Institute, the DOCS4DOCS® (D4D) clinical messaging service is provided by the Indiana Health Information Exchange (IHIE). The service receives laboratory, transcription, and radiology information from participating data sources (e.g., hospitals, laboratories) via real-time, secure Health Level 7 (HL7) feeds.[15] D4D converts the computable clinical results into a standardized reporting format that includes a header with the sending organization's logo and contact information. Once converted, the report is delivered to the intended provider. To ensure that all messages are successfully delivered, IHIE personnel follow-up on any non-fax message left unopened after 72 hours.

The DOCS4DOCS $®$ service also delivers electronic copies of discharge summaries, operative notes, and electrocardiograms (EKGs). As of February 18, 2009, there were 3,636 practices with just over 10,000 physicians utilizing the DOCS4DOCS ${ }^{\circledR}$ service throughout Indiana. Of those practices, 2,335 (64\%) received messages via fax, 1,337 (37\%) viewed messages via the webbased inbox, and 52 (one \%) accessed their messages from within their EHR system.

\section{Public Health Alerting}

In addition to delivering clinical data, a clinical messaging service can act as an adjunct to existing public health alert network functions.[16] In 2008, the Regenstrief Institute, Indiana University School of Medicine, IHIE, and the Marion County Health Department (MCHD) collaborated to develop a public health alerting interface within the DOCS4DOCS® service. The interface allows public health alerts to be delivered in a manner consistent with the physicians' existing message delivery preferences. Messages can be sent to all physicians within a jurisdiction, or messages can be targeted to specific practices based on clinical specialty (e.g., family physician, pediatrician) or geography (e.g., zip code). Figure 1 shows a screenshot of an actual alert delivered to a fictitious user's inbox. 
Development and Assessment of a Public Health Alert Delivered through a Community Health Information Exchange

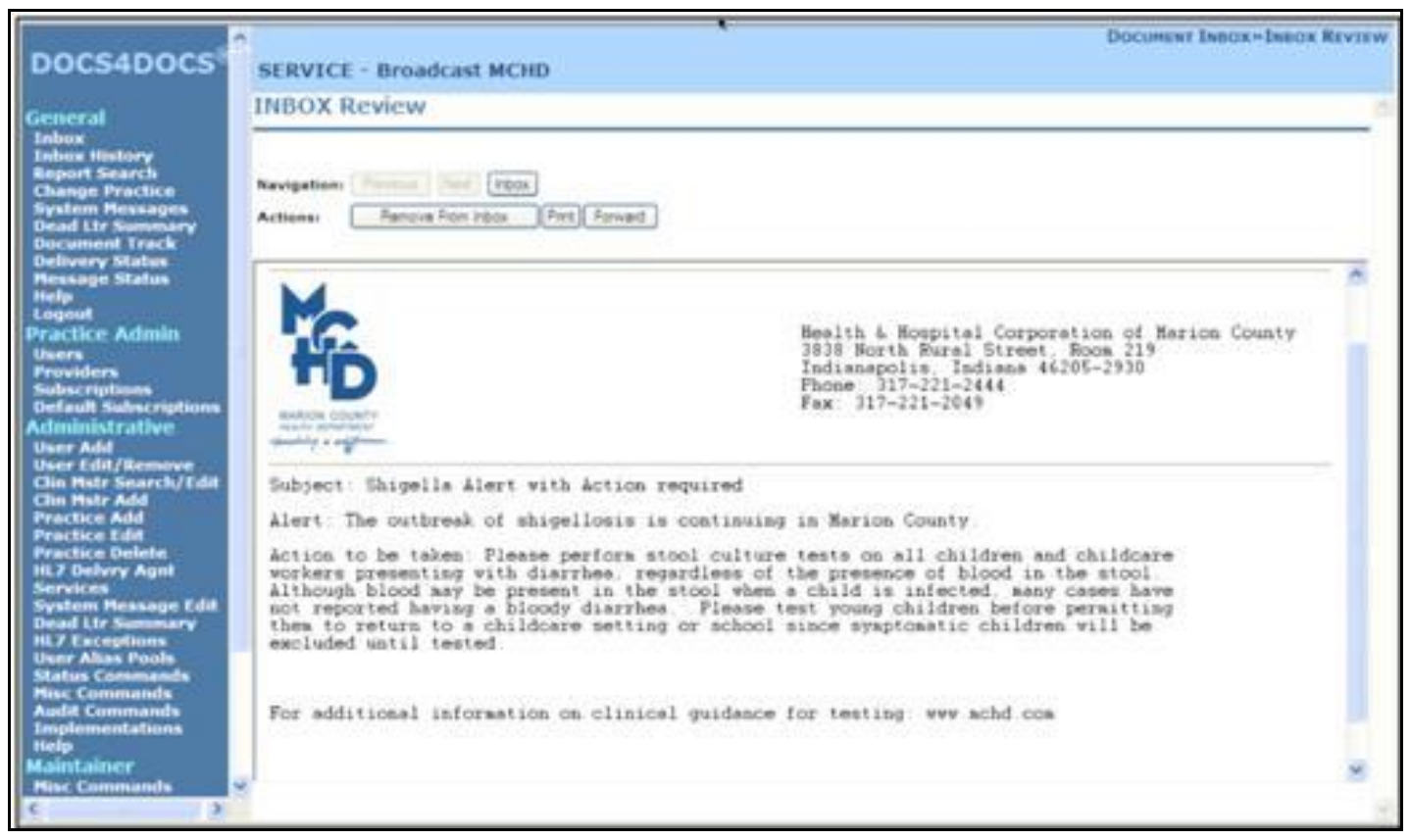

Figure 1. Physician's screen showing a public health alert sent to a Docs4Docs $®$ web-based inbox. This alert describes action to be taken for a Shigella outbreak.

\section{Alerting Workflow}

After creating the alert message, the public health agency emails it to the Regenstrief Institute where personnel, with access to the public health alerting interface, submit the message. The clinical messaging system delivers the public health alert to physicians specified in the alert. The early alerting activity has focused on the Indianapolis Metropolitan Statistical Area including Marion County. In 2009, the United States Census estimated Marion County's population at 891,000. As the state capital and Indiana's largest city, Indianapolis is the 14th largest city in the United States. MCHD is one of only two local health departments in Indiana to conduct syndromic surveillance[17] with the state and provides a variety of public health services related to population and environmental health.

\section{Syndromic Surveillance}

To conduct syndromic surveillance, MCHD utilizes two systems: the Public Health Emergency Surveillance System (PHESS)[18] and the Electronic Surveillance System for the Early Notification of Community-based Epidemics (ESSENCE). Both systems are supplied data from 74 Indiana hospital emergency departments in real-time and managed by IHIE. In monitoring the magnitude of the H1N1 flu outbreak, state and county epidemiologists monitored the count of influenza-like illness (ILI) cases.

\section{Results}

The public health alert interface within DOCS4DOCS® was completed in early 2009. Since then, nine public health alerts have been disseminated to physicians in Marion County. Table 1 


\section{Development and Assessment of a Public Health Alert Delivered through a}

Community Health Information Exchange

provides a timeline of the various alerts distributed via the DOCS4DOCS® service. Three of the alerts provided updates regarding the $\mathrm{H} 1 \mathrm{~N} 1$ outbreak of 2009. The remaining alerts distributed information regarding local outbreaks and updates concerning public health policies.

Table 1. Summary of Alerts sent by the Health Information Exchange for the Local Public Health Agency

\begin{tabular}{ll}
\hline Date Alert Sent & Description of the Alert \\
\hline $4 / 29 / 09$ & H1N1 Flu Alert \\
$5 / 13 / 09$ & H1N1 Flu Alert (follow-up) \\
$8 / 26 / 09$ & Syphilis outbreak alert \\
$9 / 15 / 09$ & H1N1 Flu vaccination information \\
$2 / 17 / 10$ & Rabies information and treatment update \\
$4 / 1 / 10$ & New vaccination requirements for school \\
$4 / 15 / 10$ & Syphilis outbreak reminder \\
\hline
\end{tabular}

On April 29, 2009, MCHD sent a H1N1 flu alert to Marion County physicians who utilize the DOCS4DOCS $®$ service. Using their syndromic surveillance system, MCHD identified a steep rise in $\mathrm{H} 1 \mathrm{~N} 1$ chief complaints and influenza testing (figure 2) and wanted to provide physicians with information on how to handle suspected cases. A subsequent H1N1 flu alert was sent on May 13, 2009, when a school closed due to a dramatic rise in H1N1 cases within the county, and a H1N1 flu vaccination information alert was sent on September 15, 2009, informing physicians on how to ensure their practice had vaccines on hand for the impending flu season.

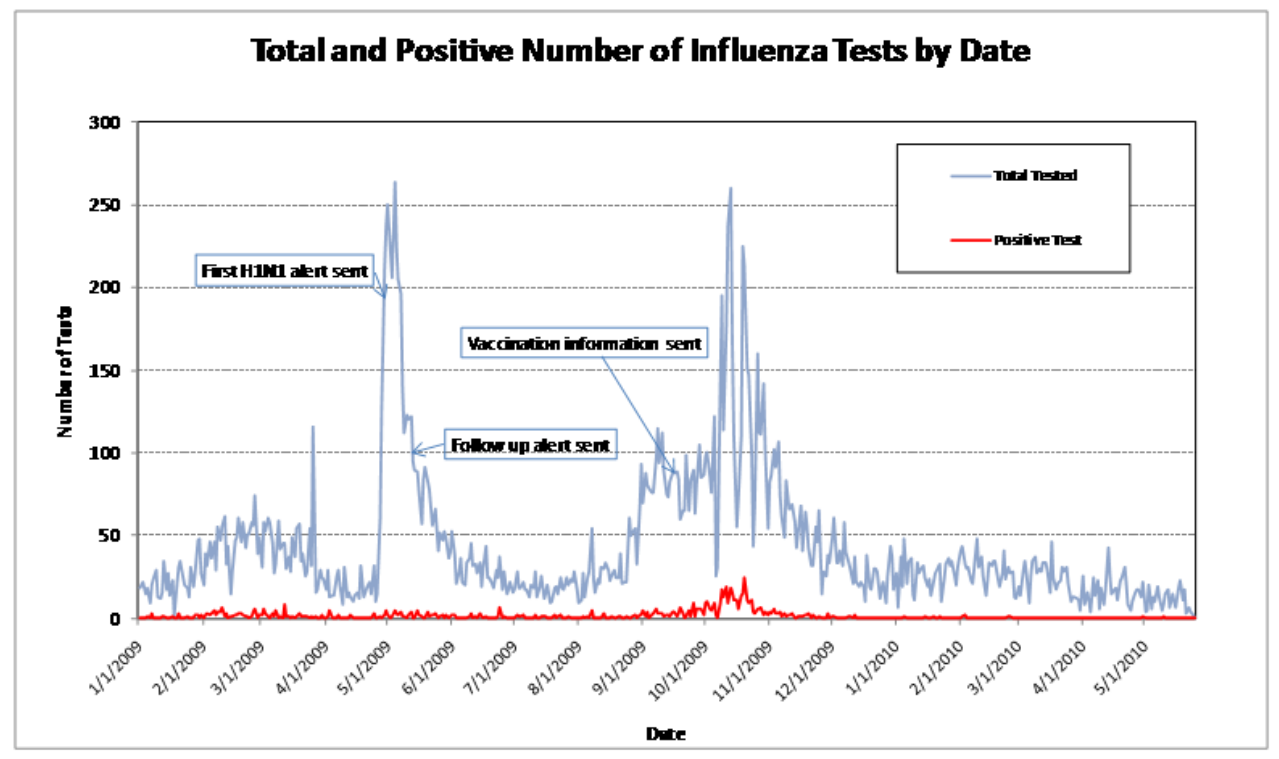

Figure 2. Laboratory testing for H1N1 during the flu season by date 


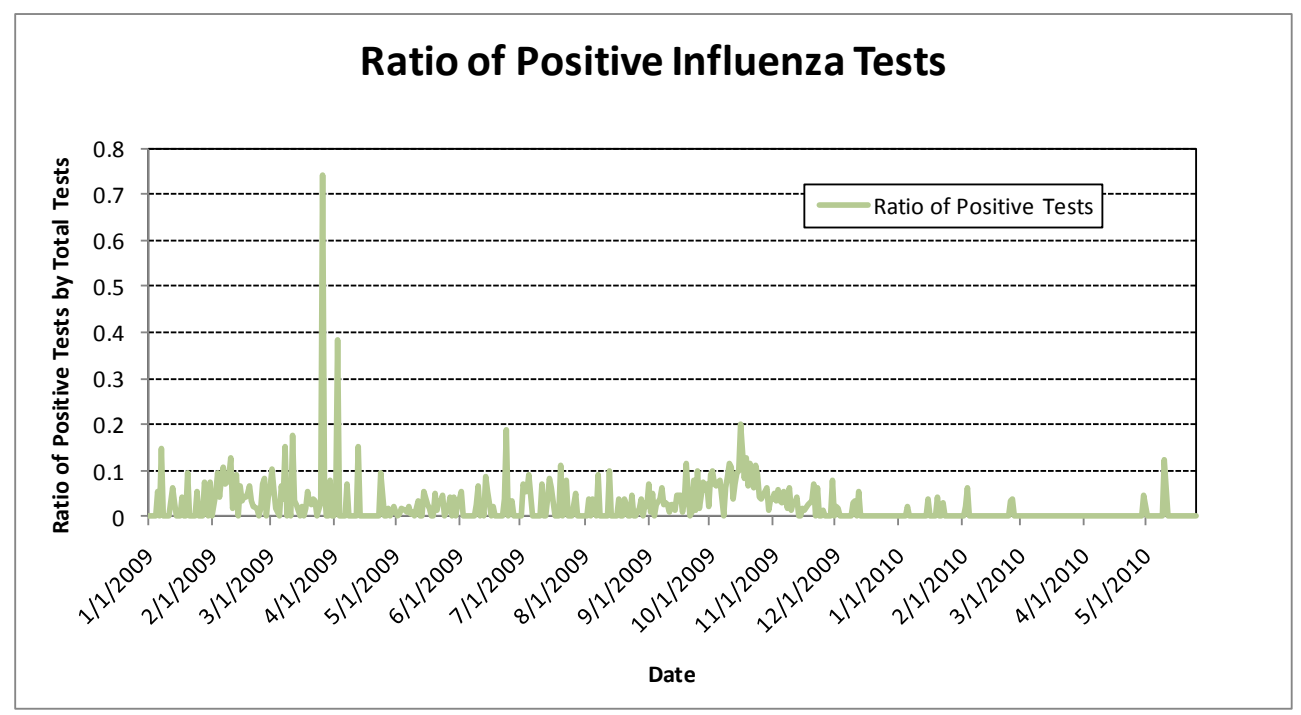

Figure 3. Ratio of Positive Influenza Tests by Date

Since January 2009, MCHD has reported an outbreak of syphilis in Marion County, Indiana. A syphilis public health alert was sent on August 26, 2009, to inform physicians of the ongoing outbreak. Since the syphilis outbreak was unresolved in early 2010, another public health alert was sent on April 15, 2010. Information about treating individuals who may have rabies was sent on February 17, 2010. In addition to public health alerts related to diseases, MCHD sent an alert on April 1, 2010 notifying physicians about new school vaccination requirements.

There were 3,085 physicians eligible to receive an alert through DOCS4DOCS® as of April 29, 2009. Of those, DOCS4DOCS® successfully processed messages for 3,021 (97.9\%) providers. Messages to 64 providers were "lost" during the message generation process. This bug was fixed prior to the sending of the second broadcast message. Of the 3,021 providers, $158(5 \%)$ of them were returned as undeliverable.

In an effort to reduce the laboratory testing burden, on April 30, 2009 the Indiana State Department of Health transmitted an alert (through the state's HAN system) providing new guidelines for sample testing of the "worried well" during the early phases of the H1N1 flu outbreak.[19] Data from the HIE-based influenza surveillance system points to a decrease in the number of samples tested after the alert was sent (figure 3). This suggests the potential impact of the electronic alerts in changing provider behavior in a short time frame for an issue of large impact on the health of the community. 
Development and Assessment of a Public Health Alert Delivered through a Community Health Information Exchange

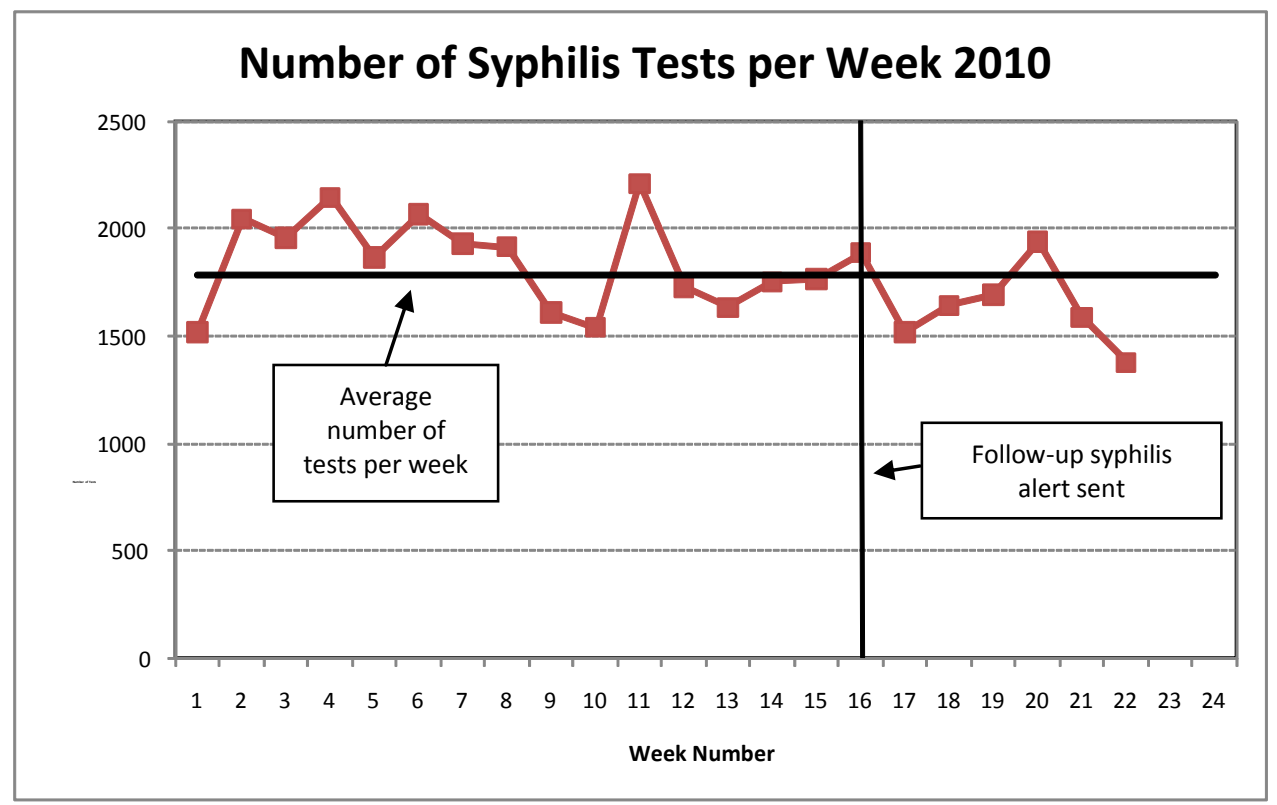

Figure 4. Number of requested syphilis tests per week during 2010.

On April 15, 2010, MCHD sent an alert describing a syphilis outbreak in the community. A review of the HIE-based data for the number of syphilis tests ordered during this period did not show a significant increase in the number of tests ordered after the April 15 date. In fact, after the first week the number of tests requested for syphilis showed signs of a decrease. A graph of this data is presented in figure 4. (The horizontal line on the graph represents the average number $(1,787)$ of syphilis tests ordered per week based on the first 23 weeks of 2010.)

\section{Discussion}

We developed and implemented an integrated alerting service for public health agencies to communicate more effectively and efficiently with clinicians. Our experience suggests that electronic public health alerts, when integrated into existing clinical workflows, have the potential to improve public health practice and clinical decision-making. Although assessment of the new service was limited, there are many potential benefits of this kind of service to public health, providers, and HIEs.

\section{Value of alerts}

There are several areas of added value through the process of sending public health alerts through the Health Information Exchange in the community. One such value is improved timeliness: the alert is delivered to the physician the same day that it is sent. With more timely information the clinical delivery system may be more likely to respond to the inciting event and potentially reduce some of the resource burden to the community.[20]

If only one organization is maintaining a list of electronic addresses for providers and the list is used by multiple organizations, the total cost for this list maintenance is reduced. There should 


\section{Development and Assessment of a Public Health Alert Delivered through a Community Health Information Exchange}

also be an additional added value for the HIE in identifying bad addresses earlier in the process and prevent the delay of sending urgent clinical data to the provider in a timely fashion.

With enhanced information about the provider, such as practice location and practice specialty, alerts from public health could be targeted to those providers that would most benefit from a community alert. Examples of targeted messages could be for outbreaks in a particular geographic location or information targeted to providers that deal in primary care or pediatrics.

Public health agencies stand to receive several benefits from an automated alerting system through a community HIE. First, agencies can improve the speed in which alerts reach clinicians. Whereas the United States Postal Mail can take several days, electronic messages through an HIE can be transmitted to clinicians within minutes. Second, the messages are received by providers through established work patterns. Third, the delivery and receipt of the messages can be monitored to confirm delivery and more quickly identify communication errors. Finally, there is a direct cost savings to the public health agency when using an HIE compared to a paper-based system.

The estimated costs for a public health alert mailing are based on previous public health mailings that occurred in the state over the last several years. The costs for envelops, letterhead, and labels were obtained from our purchasing department. The costs listed are per 100 items to make the amounts easier to read (i.e., no fractional cents are listed). The total amount of labor time to order these materials is based on placing and validating the order. The total time required for placing the labels on the envelops, printing the labels, stuffing envelopes and adding postage is based on prior mailings that averaged approximately twenty hours of total staff time for 2,000 mailed items. The distribution among the categories listed is an estimate, however; the total time is reliable and based on several mailings that occurred over the last several years. The postage cost is a savings based on the United States Postal Service listing and is a generally used business practice for larger mailings. Finally, the indirect rate is an estimate of actual indirect costs (electric, rent, etc.) and not the indirect rate that is negotiated for grants and contracts. All of these numbers are presented so that public health departments can use their own estimates if they wish to calculate an estimated savings from their own experience. The total cost savings for the public health agency in this study was estimated to be $\$ 3,638$ for each set of alerts sent. This dollar amount was calculated based on sending 3,085 alerts to providers and community. 
Table 2. Potential cost and labor benefits for Public Health by using electronic alerts ${ }^{\text {a }}$

\begin{tabular}{|c|c|}
\hline Description & Estimated Cost (per 100 letters) \\
\hline Cost of letterhead $^{\mathrm{b}}$ & $\$ 16.67$ \\
\hline Cost of envelopes (pre-printed return address) ${ }^{\mathrm{c}}$ & $\$ 16.13$ \\
\hline Cost of labels ${ }^{d}$ & $\$ 2.00$ \\
\hline Labor cost of supply ordering ${ }^{\mathrm{e}, \mathrm{f}}$ & $\$ 6.25$ \\
\hline Labor cost of printing and affixing labels ${ }^{e, g}$ & $\$ 8.33$ \\
\hline $\begin{array}{l}\text { Labor cost of stuffing envelopes and adding } \\
\text { postage }^{\mathrm{e}, \mathrm{h}}\end{array}$ & $\$ 16.67$ \\
\hline Postage cost ${ }^{i}$ & $\$ 41.40$ \\
\hline Indirect $\operatorname{cost}^{j}$ & $\$ 10.47$ \\
\hline Estimated Savings & $\$ 117.92$ \\
\hline
\end{tabular}

Notes

a. The cost of writing the alert is not included since the alert would be written and approved for either process

b. 1500 sheets/box at $\$ 250$ per box

c. $1500 /$ box at $\$ 242$ per box

d. 1500 labels package at $\$ 30$ per package

e. Assume labor and fringe at $\$ 25 / \mathrm{hr}$

f. Estimated at 15 minutes per 100

g. Estimated at 20 minutes for printing one hundred labels and affixing labels to envelopes

h. Estimated labor of 40 minutes for 100 letters

i. Commercial presorted rate from the United State Postal Service at $\$ 0.414$ per letter

j. The indirect rate is estimated to be $33.5 \%$ on labor costs only

Improving the speed of delivery of public health alerts and ensuring their integration into clinical workflow increases the likelihood that public health agencies can influence clinical decisionmaking. If providers are able to access timely, actionable information through existing work practices, there is a better chance they will modify standard care in response to the new information. Previous research has shown that providers do respond to public health alerts.[2123] For example, a community alert was sent out to providers, which established refined protocols for testing for the H1N1 virus. These testing criteria included specific information about patient symptoms in order to be tested for this virus. The provider community, adjusted the testing criteria, based on these protocols. We observed this same change in behavior when MCHD used the DOCS4DOCS® system to send an H1N1testing alert to providers.

In comparison, surveillance data following the broadcast alert describing an increase in syphilis in the community did not reveal any evidence of change in provider behavior based on a change in the number of tests requested for syphilis following the last broadcast alert. There are several reasonable explanations to be explored in order to fully understand why no effect was seen in this instance. First, the broadcast alert sent out in 2010 was a follow-up reminder from a previous alert sent out in 2009. Also, information about the outbreak was provided to the physician community through many professional channels, including other public health agency announcements and communications through supplementary professional channels. Therefore, providers may have already changed their behavior and consequently no significant increase in syphilis testing rate would be detected in the physician practice. 


\section{Development and Assessment of a Public Health Alert Delivered through a}

Community Health Information Exchange

Additionally, many of the symptoms for syphilis remain undisclosed by the patient or undetectable on physical exam. If a potential syphilis patient presents at a clinic or office with chief complaints for a condition unrelated to a potential syphilis case, the encounter may not trigger a consideration by the physician to order a syphilis test for this individual. In order for providers to better respond to this type of public health alert, a more specific epidemiological profile may be needed to notify the provider community about patients that present at the encounter with chief complaints unrelated to underlying syphilis symptoms.

Another possible explanation would be if the patient demographics shifted over the period between the two public health alerts, particularly in consideration of the change in the economic climate or other social factors. Many of these cases initiate from the county hospital. If there was a change based on the downturn in the economic conditions in the community, there would be an expected increase in patient volume at the county hospital. Therefore, there would also be an expected increase in the number of patients screened for syphilis testing.

We considered looking at a change in rates for the analysis of this parameter; however, determining the denominator for this analysis was difficult. There is only an average of 1,787 syphilis tests ordered per week and approximately 2.7 million messages over this same period. Therefore, the calculated rates would be small and any change hard to measure. Once again, a more specific epidemiological profile would help in this analysis as well. It is clear that further study is necessary in order to fully assess and understand the ability of public health broadcast alerts to impact physician behavior.[24]

Providers also stand to benefit from electronic public health alerts delivered through a community HIE. First, providers will see a reduction in the postal mail they receive from public health. Second, the information sent from public health will be timelier, potentially improving clinicians' perception of public health agencies. Finally, the information arriving in the alerts will be actionable and provide tailored guidance that clinicians can use to enhance the quality and safety of care they deliver to patients. Preliminary conversations with providers in our region reveal that many clinicians welcome this kind of change in how public health communicates with the provider community.

The community HIE also benefits from this partnership. This activity provides an additional service to a common platform and provides another mechanism for validating and verifying provider addresses in the community. This updated provider information is important when sending out clinical information regarding patients in the provider's practice. Since the infrastructure for delivering clinical health information is being reused for the delivery of broadcast alert messages, total costs for the community are potentially reduced. Finally, information concerning the contact lists for physicians is necessary for both public health and the community health information exchange. Since this information is collected only once and maintained by one organization, total costs are reduced.

The overall community also benefits from this partnership. Since information about community outbreaks are provided in an manner that are easily maintained and delivered through a timely mechanism, the disease burden experienced by the community should be reduced. This change 


\section{Development and Assessment of a Public Health Alert Delivered through a}

Community Health Information Exchange

should result in a more effective response to community acquired infections, and therefore a more effective use of healthcare resources.

\section{Future Directions}

End-user acceptance of this new data sharing mechanism (broadcast messages) will be strongly influenced by end-user perception of the process. Consequently, the attitude of physician and public health groups to how these new data sharing mechanisms are perceived will be evaluated. We will also examine if there is a difference of perception or practice by physicians that are primarily paper-based in the office versus those physicians are using a clinical messaging system like Docs4Docs. Each of these outcome measures may be evaluated for a survey tool to gain the perspective of the position and may be measured empirically through information gained in the clinical data repository. We plan to evaluate the perceived utility of these interventions with stakeholders from both public health and clinical health care settings to gage unforeseen complexities and advantages. Finally, because general alerts seem to be less effective in producing behavioral changes in the provider practice, future work to identify what types of detailed information included in the public of alert are most likely to produce provider changes is warranted.

\section{Conclusions}

The process of providing public health alerts through the community health information exchange provides a cost savings to public health over the traditional system of a mail-based public health alert. More analyses are needed to fully assess the other impacts of this type of delivery system to include provider groups and the health information exchanges.

Delivering public health alerts to physicians using an existing clinical messaging system represents a well-scoped and incremental step forward that has the potential to improve process efficiency, including reduced costs and improved timeliness. By engaging both clinical stakeholders and public health stakeholders in such a pervasive and ongoing activity, we can continue to build the digital bridge and trust relationships between the two domains that are necessary to establish the infrastructure for the next stage of more complex public health decision support processes.

\section{Acknowledgements}

This work was funded by the Centers for Disease Control \& Prevention under contract 2002008-24368. The content of this publication does not necessarily reflect the views or policies of the Department of Health and Human Services, nor does mention of trade names, commercial products, or organizations imply endorsement by the U.S. Government.

The views expressed in written conference materials or publications and by speakers and moderators at HHS-sponsored conferences, do not necessarily reflect the official policies of the Department of Health and Human Services; nor does mention of trade names, commercial practices, or organizations imply endorsement by the U.S. Government. 


\section{Development and Assessment of a Public Health Alert Delivered through a}

Community Health Information Exchange

\section{References}

[1] Thacker, S.B. and R.L. Berkelman, "Public Health Surveillance in the United States. Epidemiology Review”, 1988. 10: p. 164- 190.

[2] CDC. Health Alert Network. 2010; Available from:http://www2a.cdc.gov/han/Index.asp.

[3] Daniel, J.B., et al., Connecting health departments and providers: syndromic surveillance's

last mile. MMWR Morb Mortal Wkly Rep, 2005. 54 Suppl: p. 147-50.

[4] Baker, E.L. and J. Porter, The health alert network: partnerships, politics, and preparedness. J Public Health Manag Pract, 2005. 11(6): p. 574-6.

[5] AHRQ. Health Information Exchange. Key Topics 2009 February [cited 2010 February 7]; Available from: http://healthit.ahrq.gov/hie.

[6] Dixon, B.E., A. Zafar, and J.M. Overhage, A Framework for evaluating the costs, effort, and value of nationwide health information exchange. J Am Med Inform Assoc, 2010. 17(3): p. 295301.

[7] Dixon, B.E. and S. Scamurra. Is there such a thing as healthy competition? in Annual HIMSS Conference \& Exhibition. 2007. New Orleans, LA: HIMSS.

[8] Hessler, B.J., et al., Assessing the relationship between health information exchanges and public health agencies. J Public Health Manag Pract, 2009. 15(5): p. 416-24.

[9] Overhage, J.M., S. Grannis, and C.J. McDonald, A comparison of the completeness and timeliness of automated electronic laboratory reporting and spontaneous reporting of notifiable conditions. Am J Public Health, 2008. 98(2): p. 344-50.

[10] Grannis, S., et al., The Indiana Public Health Emergency Surveillance System: ongoing progress, early findings, and future directions. AMIA Annu Symp Proc, 2006: p. 304-8.

[11] Grannis, S.J., et al., How disease surveillance systems can serve as practical building blocks for a health information infrastructure: the Indiana experience. AMIA Annu Symp Proc, 2005: p. 286-90.

[12] Rosenman, M., et al., Computerized reminders for syphilis screening in an urban emergency department. AMIA Annu Symp Proc, 2003: p. 987.

[13] Dexter, P.R., et al., A computerized reminder system to increase the use of preventive care for hospitalized patients. N Engl J Med, 2001. 345(13): p. 965-70.

[14] Dexter, P.R., et al., Effectiveness of computer-generated reminders for increasing discussions about advance directives and completion of advance directive forms. A randomized, controlled trial. Ann Intern Med, 1998. 128(2): p. 102-10.

[15] Barnes, M., Lessons learned from the implementation of clinical messaging systems. AMIA Annu Symp Proc, 2007: p. 36-40.

[16] Grannis, S., P.G. Biondich, and B.W. Mamlin, How Disease Surveillance Systems Can Serve as Practical Building Blocks for a Health Information Infrastructure: The Indiana Experience. AMIA Annu Symp Proc, 2005.

[17] Grannis, S., et al., The Indiana Public Health Emergency Surveillance System: Ongoing Progress Early Findings and Future Directions. AMIA Annu Symp Proc, 2006.

[18] Gamache, R.E. and M. Wade, The Indiana Public Health Emergency Surveillance System (PHESS), in eHealth Initiative, Blueprint Award for Improving Population Health. 2007:

Washington, DC.

[19] Health, I.S.D.o., Indiana Epidemiology Newsletter, 2009. 17(4). 


\section{Development and Assessment of a Public Health Alert Delivered through a}

Community Health Information Exchange

[20] Elson, R.B. and D.P. Connelly, "Computerized Patient Records in Primary Care: Their Role in Mediating Guideline- Driven Physician Behavior Change. Archives of Family Medicine, 1995. 4: p. 698-705.

[21] Marc Rosenman, M., et al., Computerized Reminders for Syphilis Screening in an Urban Emergency Department. AMIA Annu Symp Proc., 2003. 2003.

[22] Gamache, R.E., The Indiana Medical Error Reporting System, in Indiana HealthCare Executives Network. 2006: Indianapolis, IN.

[23] Staes, C.J., et al., Computerized Alerts Improve Outpatient Laboratory Monitoring of Transplant Patients. Journal of the American Medical Informatics Association, 2008. 15(3): p. 324-332.

[24] The purpose of first syphilis broadcast alerts that were sent through the community HIE were to determine the feasibility of the HIE to send these types of messages. The measurement of the impact of these messages was clearly a greater priority after the feasibility was demonstrated. The associated attributes for messages sent after the initial messages are clearly more aligned to measure outcome in the provider community. 